\title{
Lean and Automate Manufacturing and Logistics
}

\author{
Bernardo Nicoletti \\ Master in Procurement, Università di Roma Tor Vergata, Rome, Italy \\ info@bernardonicoletti.com
}

\begin{abstract}
This paper presents the Lean and Automate method on how to streamline and automate simultaneously the production and logistics processes in order to improve quality, reduce waste, and increase agility using Lean Six Sigma with the support of some advanced technologies. The approach is based on research which showed that for the best application of Lean Six Sigma to production and logistics, the new processes should be improved taking into account also the possible use of advanced automation. The case studies provide good practical examples of the applications and implications of advanced technology in production, with theoretically grounded insights. The findings suggest that Lean and Automate could be a key enabler of Lean Six Sigma in the manufacturing organizations.
\end{abstract}

Keywords: Lean Six Sigma, Production, Logistics, Automation, ICT.

\section{Introduction}

The economic crisis has pushed the organizations to become more agile and leaner, in order to adapt rapidly to changing socio-economic conditions. The re-engineering of production and logistics processes must take into account this imperative. Lean Six Sigma combines the better of two distinct methods [1]: Six Sigma, which helps in reducing the number of defects and the variation of the outputs, and Lean Thinking which helps in reducing the cycle times. A certain number of organizations have achieved excellent results by the use of these methods in different fields. There is another extremely important aspect in the optimization of the processes not included in this approach, which is the flexible integration of the processes and their activities. The lean approach includes the principle of flow. The recipe to achieve flow is to balance the work in the process. This approach often assumes a constant production. It becomes a hard coded integration.

Lean and Automate aims at the same time to streamline and automate the production and logistics processes and make them agile and lean at the same time. Out of a long experience in different types of organizations and several research works in production and logistics process design and management and tools and digitization techniques [2], this approach shows clearly the sequence of activities to integrate the methods of business process improvement and automation in any production and logistics organization. 


\section{Literature Review}

Lean Six Sigma includes a set of principles and tools that assist in the identification and steady elimination of waste $(M u d a)$ and the reduction of defects and variability in the output of a process Quality and costs in production and logistics are strategic elements [1], Flexibility is paramount in modern times. There is the need to use in the world of production and logistics the flexible automation technologies, now more and more available at lower costs, applying them to re-engineered processes.

Lean Six Sigma implementations provide several benefits, among them: cost reduction, productivity increase, quality improvement, lead time reduction, supplies reduction, and customer satisfaction improvement. Lean Thinking has been applied in the production context through Lean Production studies, However, these studies do not take into account new technologies more and more available. Actually, Sugimori et al. argued that the use of the automation for production introduces unnecessary costs, overproduction, and uncertainty [3]. This theory contrasted with the trends since the 80's, when the interest on MRP and ERP systems, numerical control machines, and fully-automated production lines has been huge. The highly automated organizations were less vulnerable to the typical problems of manual work. However, there were examples of over-investment in automation that have worsened the flexibility and the ability to respond to the demand changes (General Motors in the'80s; CIM),

The automation alone to improve performance remains uncertain [4]. This is the reason why the automation must be flexible and connected with an improvement in the processes. Powell and Skjelstad [5] applied a specific sensor technique (RFID) to the manufacturing processes. They presented a method and two business cases which proved the usefulness of this type of advanced technology in manufacturing companies.

This paper is a descriptive study [6]. Inductive reasoning is used to propose a conceptual framework for the use of advanced technology as an enabler in the development and further support of the Lean Six Sigma approach. The primary research method deployed is multiple case study research. The scientific foundation for the research is the literature from the production and logistics management with a focus upon the advanced automation technologies [7] and [1].

\section{The Lean and Automate Method}

The Lean and Automate method can be divided into six macro phases and 20+1 micro phases (see Fig. 1) ${ }^{1}$. The 21 st phase is optional.

\section{Macro Phase 0: Preliminary}

- Context: Identify the requests of the stakeholders and the EHS;

- Culture: Detect the culture of the organization and of the community;

- Vision: Tackle the effectiveness, efficiency, economy, quality, and flexibility;

- Strategy: Define the processes to be improved and the plans;

\footnotetext{
${ }^{1}$ This is a modified version of the Lean and Digitize method presented in [1] to take into account the peculiarities of the manufacturing environment.
} 


\section{Macro Phase 1: Define and Measure}

- Kick-off: Launch the project and notify the stakeholders;

- Governance: Define who and how should manage the project; set up the Team;

- Voice of the Customer: Listen to the Voice of the Customers (Voc);

- Metrics: Translate the VoC in Critical-to-Quality (CtQ) factors;

- As-Is: Map the existing process;

\section{Macro Phase 2: Analyze and Process Design}

- Lean: Define how to improve the process during workshops;

- Kaizen Plan: Define the improvement intervention plan.

\section{Macro Phase 3: Architecture Design}

- Architecture Design: Define the process and the automation architecture;

\section{Macro Phase 4: Build, Test and Deploy}

- Build and Test: Implement and test the chosen solution;

- Change management: Manage the transformation;

- Deploy: Implement the chosen solution;

- Documentation: Issue/update the documents of the new automated processes;

\section{Macro Phase 5: Verify}

- Verify: Control the improvements,

- Benefits: Assess the external and internal benefits;

- Lessons Learned: Learn from the initiative;

- Celebration: Acknowledge the team's work;

\section{Optional Macro Phase 6: Replicate in Similar Situations}

- Rollout: Replicate the solution to the different departments.

For the purpose of continuous improvement, once the project is completed, one must use DMAIC. which typically leads to the need of a technological change.

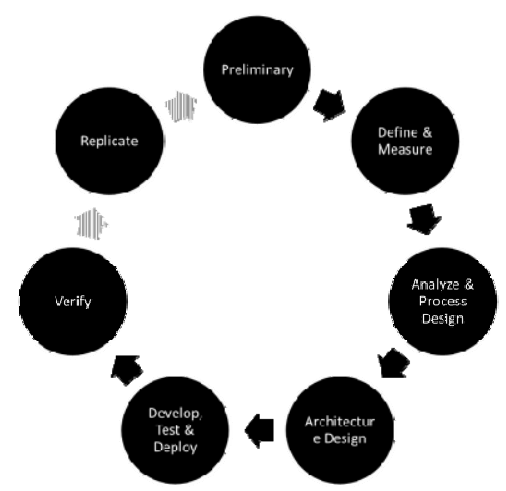

Fig. 1. The Lean and Automate Method 
The organizations must treat the initial Lean and Automate project as the beginning of an iterative cycle that generates continuous improvement. Process improvement should not be triggered by a problem, but be part of the organizational culture.

\section{The Changing Environment}

A research on the trends in production showed that one of the most relevant aspects for improvement is the technological optimization of processes ${ }^{2}$. Modern technologies improve the efficiency of processes. The manufacturers will buy more and more equipment to save costs and optimize business processes.

We state that it is important to move from the automation of machines to the automation of processes. Machining centers are sophisticated Computer Numerically Controlled machines that can perform milling, drilling, tapping, and boring operations at the same location with a variety of tools. They are still isolated. An important development is to automate the full process rather than just one of its components. In order to reach this goal, it is essential to integrate different machines or entire sectors of the production cycles. This paper concentrates on an architecture based on four technologies that can help on this respect: The Internet of Things (IoT), Sensor technologies, either with bar codes or RFID; Big Data; and Business process intelligence.

The following sections define each one of these technologies and analyze each one of them and their possible contribution to process improvement. Their implementation should be done according to several generations, implementing one technology at a time and using some generalized middleware for managing interfaces in a more standardized way ${ }^{3}$.

\subsection{IoT}

Internet of Things (IoT), also called Machine to Machine (M2M), refers to technologies that allow both wireless and wired systems to communicate with other devices. Such communication can be accomplished by having a remote network of machines relay information back to a central hub for analysis. Normally, a multi-tier architecture is used to exchange information at lower levels

IoT when combined with Internet and with cloud computing is an excellent way to add flexibility and investment saving to the collaboration also with vendors [8]. A variation to IoT is to use a ZigBee network.

\subsection{RFID}

Radio-frequency identification (RFID) is the use of a wireless non-contact system allowing radio-frequency electromagnetic fields to transfer data from a tag attached to an object. The purpose is automatic identification, possible storing of data, and

\footnotetext{
2 http://en.wikipedia.org/wiki/ Productivity_improving_technologies_(historical)

${ }^{3}$ One example of standard for interfaces is for instance XLM and OAGIS, as powered by OAGi (http: / / www . oagi .org/dnn2 /, accessed 10 March 2013).
} 
tracking. Unlike a bar code, the tag does not need to be within line of sight of the reader. It may be embedded in the tracked object. An RFID tag attached to a product during its manufacturing can be used to track its progress through the production line. In the last few years, three key factors drove a significant increase in RFID usage: decreased cost of equipment and tags, increased performance to a reliability of $99.9 \%$, and improved international standards [9].

\subsection{Big Data}

Big Data is a collection of data characterized by high volume, variety (structured and unstructured), and velocity (in terms of speed of access) so complex that it becomes difficult to process using traditional database management tools or data processing applications.

Data sets grow in size in part because they are increasingly being gathered by information-sensing mobile devices, aerial sensory technologies (remote sensing, for instance RFID), software logs, video, cameras, microphones, radio-frequency identification readers, and wireless sensor networks.

\subsection{Business Process Intelligence}

Business Process Intelligence (BPI) is an innovative approach focused not only on measuring and reporting but also on managing the processes. through their data and workflows, differently to traditional Business Intelligence.

BPI supports also analytics, with production of statistics, reports, and dashboards. They allow a better visibility of the processes. For example in the case of production, the analytics tools allow analyzing the KPI and help take actions.

\subsection{Flexible Integration Technology}

As sensor technology and network technology develop, a computer system (or a machine) is becoming able to collect information about the real world by communicating with other machines without any help from human beings. In conventional IoT systems, servers for collecting and analyzing information from machines were developed individually. Now, virtualization and cloud technologies have enabled the IoT cloud or ZigBee network, in which all the functions such as communication with various devices and the collection, storage, and use of Big Data and BPI can be managed centrally. These clouds have the following features:

(1) Data collection and storage: An IoT cloud can communicate with any devices, collecting, storing data, and managing devices. A combination of multiple data sources can be analyzed easily thanks to a large amount of information available;

(2) Data analysis: The Big Data allows performing statistical calculations;

(3) BPI can operationalize the decisions in a flexible way.

An example of the integrated solutions can be the use of a e-Kanban to replace the Kanban cards with RFID tags. This allows to fully automating the 'identification' of the status of the processes, with greater reliability and without operator intervention [10] and [11]. E-Kanban can reduce the levels of inventories of work in progress (WIP), maintain tight control, and integrate with suppliers. 
Another application is to use automated sensors, wireless networks and Big Data to update digital billboards. This allows organizations to implement what is called Andon and allows quick changes for messages [1]. The system may include the stop of production so the issue can be corrected. Some modern alert systems incorporate audio alarms, text, or other displays.

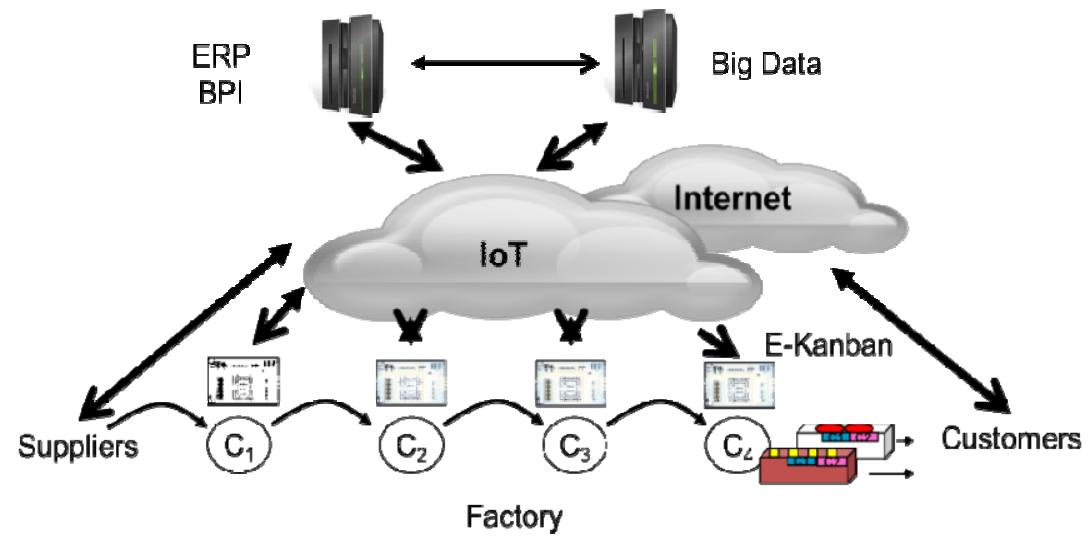

Fig. 2. The Final Architecture

The final architecture is shown in Fig. 2. It takes time to build. It is important to have the vision of where one want to arrive and then build in a multi-generation plan. Each generation will bring substantial benefits per se. The final generation brings all the components together and reaps the synergies.

\section{The Support of New Technologies to the Lean and Automation Method}

This architecture allows reaping the benefits of flexible integration. IoT, RFID, Big Data, and Business Process Intelligence introduce additional benefits per se.

- RFID can bring the benefits of automatic sensing in processes.

- IoT can improve the flexible integration of the activities in the value stream;

- Big Data can help in making the flow pulled by the customers, by allowing adding an intelligence of the demand of the customer;

- BPI can bring the benefits of the combination of Business Intelligence and the management of process workflows.

All together these technologies and the improvement of processes can add value thanks to their characteristics: Traceability, Visibility, Memory, and Positioning/ localization. Lean and Automate can improve these macro-phases of the Lean Six Sigma approach:

- Define and Measure, and Verify:

- Managing the life cycle of the components (that is tools);

o Compliance; 
- Plan of routine and non-routine maintenance;

- Identify problems related to specific lots and take immediate maintenance actions;

- Improvements in production due to an immediate measure;

- Source of statistical data for the definition of process improvement and analysis of materials;

- Identification and status changes on a component;

- Paper savings.

- Analyze and Process Design:

- Improved security by storing the relative weights, needs qualification, requirements of Quality Assurance, and so on;

○ Configuration management;

- Improved communication of critical data.

The new architecture can help especially in reducing some of the seven wastes, listed in Lean Six Sigma, such as waiting time, by the improvements in search times, waiting, and movements; reduction in repairs/errors/faults, thanks to the identification of nonstandard components, any failure or theft, and help in the analysis of the root causes; reduction in stocks, by the identification of components not good to be phased out.

\section{Case Studies}

In order to explore the practical relevance of Lean and Automate, we report a summary of two case studies, which compare the situations where advanced technology is used versus when it is not used [12]. In both cases, engineering manufacturing companies were considered with each job being different from another one, each one with either one or few items. The products were dies for aluminum extrusion. In both cases, we analyzed the management of a tool inventory with 2500 tools for ten work stations for the processing and production of hot channels for plastic materials. In one of the companies, RFID tags were applied to the tool holder cone on machining centers. BPI software was used to analyze and manage processes.

The possible inefficiencies in the tool management on the side of the machines are their possible damage, the times for the data entry and setting up the tools.

The possible inefficiencies on the side of the tools are the times to find the suitable tool, the reduction in the useful life of the tools, oversizing of the tool inventory, improper choice of the tools, and loss of historical information.

The advanced technology can impact on all the inefficiencies listed above. Quantitative benefits are the savings and the improvement in the Overall Equipment Effectiveness - OEE, a hierarchy of metrics to evaluate how effectively a manufacturing operation is utilized [13]. OEE is based on three separate but measurable components: Availability, Performance, and Quality.

Leaving aside the details of the two cases, the use of advanced technology brought the reduction in the number of tools in the inventory, in the unscheduled events and errors in the data entry; the possibility of implementing policies of tool sharing among the machines; the choice of the right tools: the reduction in the machine downtime 
doing in parallel the operations and the search of the right tools, the reading of the part program and the presetting. From a quantitative point of view, the advanced technology brought an increase in the average value of the OEE per each machining center of $27 \%$ [12].

\section{Conclusions}

Lean and Automate responds to the lack in the literature of a consistent method that manages and integrates the classical activities of streamlining production and logistics processes with the activities of flexible automation. Our research implies:

Proposition 1: Unlike the traditional production and logistics context, where the Lean Six Sigma method requires a reduction of digitization, automation is essential for the improvement of the processes, especially to make them flexible;

Proposition 2: Automate a process not streamlined is counterproductive.

The practical examples in this paper represent a starting point for the application of advanced automation to enable the deployment of Lean and Automate throughout the manufacturing and logistics processes. More examples are necessary. Further research should investigate the applicability of advanced technology for the optimization of fields such as production scheduling by using real-time information

\section{References}

1. Nicoletti, B.: Lean and Digitize. Gower Publishing, Abingdon (2012)

2. George, L., et al.: What is Lean Six Sigma. McGraw-Hill, New York (2003)

3. Sugimori, Y., et al.: Toyota production system and Kanban system. International Journal of Production Research 15(6), 553-564 (1977)

4. Cheung, C.F., et al.: An agent oriented and knowledge-based system for strategic e-procurement. Expert Systems 21(1), 11-19 (2004)

5. Powell, D., Skjelstad, L.: RFID for the extended lean enterprise. International Journal of Lean Six Sigma 3(3), 172-186 (2012)

6. Arbnor, I., Bjerke, B.: Methodology for Creating Business Knowledge. Sage, London (2009)

7. Bell, S.: Lean Enterprise Systems: Using IT for Continuous Improvement. Wiley, Hoboken (2006)

8. Nicoletti, B.: Cloud Computing in Financial Services. Palgrave, London (2013)

9. Glover, B., Bhatt, H.: RFID essentials, pp. 88-89. O'Reilly Media, Sebastopol (2006)

10. Drickhamer, D.: The Kanban e-volution, Material Handling Management (2005), http: / / www. mhmonline.com

11. Powell, D.: Radio Frequency Identification, Inform (The Institute of Operations Management) (2008)

12. Dovere, E.: Valutazione dell'efficienza delle machine utensili con parco utensili gestito tramite sistemi d'identificazione RFID, Fabbrica Futuro, Bologna (March 6, 2013)

13. Smyth, R.: Exploring the usefulness of a conceptual framework as a research tool: a researcher's reflections. Issues in Educational Research 14(2), 167-180 (2004) 